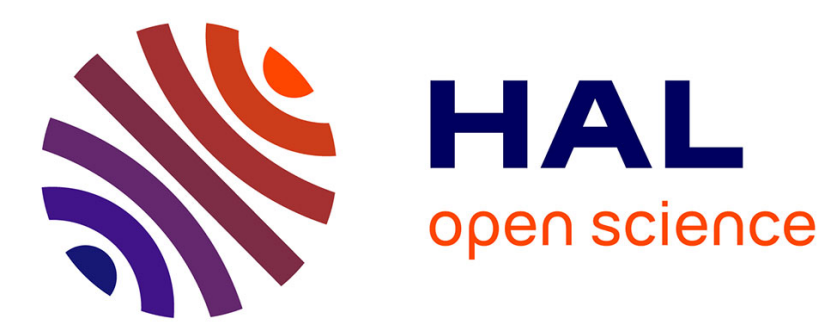

\title{
POLITIQUES PUBLIQUES ET ARTICULATION VIE PROFESSIONNELLE / VIE FAMILIALE
}

\author{
Jacqueline Heinen, Helena Hirata, Roland Pfefferkorn
}

\section{To cite this version:}

Jacqueline Heinen, Helena Hirata, Roland Pfefferkorn. POLITIQUES PUBLIQUES ET ARTICULATION VIE PROFESSIONNELLE / VIE FAMILIALE. Cahiers du Genre, 2009, 'Etat, travail, famille : 'conciliation' ou conflit', 46, pp.5-16. 10.3917/cdge.046.0005 . hal-01291910

\section{HAL Id: hal-01291910 https://hal.science/hal-01291910}

Submitted on 22 Mar 2016

HAL is a multi-disciplinary open access archive for the deposit and dissemination of scientific research documents, whether they are published or not. The documents may come from teaching and research institutions in France or abroad, or from public or private research centers.
L'archive ouverte pluridisciplinaire HAL, est destinée au dépôt et à la diffusion de documents scientifiques de niveau recherche, publiés ou non, émanant des établissements d'enseignement et de recherche français ou étrangers, des laboratoires publics ou privés. 


\section{POLITIQUES PUBLIQUES ET ARTICULATION VIE PROFESSIONNELLE / VIE FAMILIALE}

Introduction

Jacqueline Heinen,Helena Hirata, Roland Pfefferkorn

\section{L'Harmattan | « Cahiers du Genre »}

2009/1 n $46 \mid$ pages 5 à 16

ISSN 1298-6046

ISBN 9782296090682

Article disponible en ligne à l'adresse :

http://www.cairn.info/revue-cahiers-du-genre-2009-1-page-5.htm

\section{Pour citer cet article :}

Jacqueline Heinen et al., « Politiques publiques et articulation vie professionnelle / vie familiale. Introduction », Cahiers du Genre 2009/1 (n 46), p. 5-16.

DOI 10.3917/cdge.046.0005

Distribution électronique Cairn.info pour L'Harmattan.

(C) L'Harmattan. Tous droits réservés pour tous pays.

La reproduction ou représentation de cet article, notamment par photocopie, n'est autorisée que dans les limites des conditions générales d'utilisation du site ou, le cas échéant, des conditions générales de la licence souscrite par votre établissement. Toute autre reproduction ou représentation, en tout ou partie, sous quelque forme et de quelque manière que ce soit, est interdite sauf accord préalable et écrit de l'éditeur, en dehors des cas prévus par la législation en vigueur en France. Il est précisé que son stockage dans une base de données est également interdit. 


\title{
Politiques publiques et articulation vie professionnelle / vie familiale
}

\author{
Introduction
}

Les textes réunis dans ce volume questionnent les politiques publiques au regard de l'articulation vie professionnelle/vie familiale. S’agissant des femmes, depuis le début des années 1990, cette articulation ainsi que les politiques publiques la concernant sont le plus souvent présentées en termes de 'conciliation'. Mode de conceptualisation, d'une part, définition de politiques - notamment au niveau de l'Europe - d'autre part, la 'conciliation travail/famille' a produit une importante littérature tant étatique que scientifique ${ }^{1}$. Cette notion de 'conciliation', qui se présente comme universelle mais ne s’applique qu'aux femmes, a cependant, et à juste titre, fait l'objet de vives critiques (Junter-Loiseau 1999). En effet, elle "semble suggérer que le cumul des tâches domestiques et de l'exercice d'une activité professionnelle peut se faire dans des conditions harmonieuses » (Fagnani, Letablier 2001, p. 4). Des expressions alternatives ont été proposées par des chercheuses travaillant dans ce domaine qui mettent l'accent sur la contradiction, la tension ou le conflit, plutôt que sur une supposée 'conciliation'.

\footnotetext{
${ }^{1}$ Pour une présentation analytique de cette bibliographie, voir par exemple Fagnani, Letablier (2001). Pour un aperçu de l'état de la discussion aujourd'hui, à partir d'une approche par les entreprises, voir Dauphin (ed) (2008). Pour une présentation de la problématique rapports sociaux de sexe et politiques publiques, voir par exemple Gautier, Heinen (1993), Heinen (2000), et Fouquet (2005).
} 
Cependant, les institutions publiques françaises et européennes continuent à présenter les politiques sociales et familiales en termes de 'conciliation'. De plus, ces politiques sont suivies avec attention dans des pays comme le Japon, où le manque d'équipements pour la petite enfance et les politiques d'emploi mises en œuvre par les entreprises rendent particulièrement problématique la poursuite d'une activité professionnelle pour les mères. Ces contradictions expliquent en grande partie l'effondrement du taux de natalité dans ce pays. L'expression work-life balance y est utilisée pour désigner ce qui en Europe continue à être présenté comme la 'conciliation travail/famille' (Hiroki 2008). Au-delà des insuffisances en termes de politiques publiques et d'équipements sociaux, les analyses japonaises relatives à l' 'équilibre entre le travail et la vie familiale' visent aussi à interroger les normes sociales qui sont prégnantes et rendent précisément cet équilibre difficile, voire impossible, à atteindre pour les mères.

Le cas des pays du Nord de l'Europe montre une autre configuration, souvent qualifiée de 'welfare ami des femmes' en référence à la façon dont l'État prend en charge une large part des responsabilités concernant la petite enfance. Le régime de care des pays nordiques se caractérise en effet conjointement, depuis un demi-siècle, par l'existence de services extensifs en direction des jeunes enfants et des personnes âgées et par un taux d'activité professionnelle élevé chez les femmes, par contraste avec le régime méditerranéen qui, jusqu'au début des années 1990, présentait des traits inverses. Il est toutefois plus difficile aujourd'hui qu'hier de classer les divers pays dans tel ou tel régime, compte tenu d'un double processus à l'œuvre : d'une part, la garde des jeunes enfants relève davantage de l'État et se traduit par des dispositifs plus généreux qu'il y a quinze ou vingt ans dans tous les pays européens, y compris ceux du Sud de l'Europe ; d'autre part, le régime nordique a tendu à se diversifier, surtout dans le domaine des allocations financières. Il conserve toutefois des traits distinctifs de par le caractère individuel (et non familial) des droits consentis, le montant relativement élevé des prestations versées en cas de congé parental et les politiques incitatives à l'égard des pères. Ceci étant, la division du travail est loin d'être abolie dans ces pays où les 
mères continuent à assumer une part des tâches de care beaucoup plus grande que leurs conjoints et où elles prennent notamment une durée de congés parentaux nettement plus étendue qu'eux (Leira 2002 ; Williams 2004 ; Lister et al. 2007).

Les textes rassemblés ici répondent à plusieurs objectifs. Examiner, dans une optique historique, le rôle de l'État et des pouvoirs publics locaux ou régionaux quant à la configuration des rapports sociaux de sexe, dans la sphère du travail comme au sein de la famille - en lien, notamment, avec les facteurs démographiques et les fluctuations du marché de l'emploi. Analyser les diverses formes des modes de garde de la petite enfance et leurs effets contrastés pour les adultes concernés — les femmes, au premier chef — selon qu'il s'agit de services collectifs (crèches, maternelles) ou de dispositifs incitant à une prise en charge individuelle des jeunes enfants (congés parentaux de tous ordres, dégrèvements fiscaux favorisant l'emploi d'assistantes maternelles à domicile, etc.). Donner à voir divers modèles de répartition des tâches dans les activités de care (entre les hommes et les femmes et à l'intérieur du groupe des femmes) en fonction de la place assignée aux pères et aux mères, ainsi qu'en fonction des transformations économiques à l'échelle mondiale, de la polarisation Est-Ouest et Nord-Sud, et même Sud-Sud, ainsi que de l'importance et des caractéristiques des flux migratoires.

Les contextes sociétaux examinés sont variés. Ils ont trait principalement à l'Europe (Allemagne, Suisse, Espagne, GrandeBretagne, Pologne, Hongrie) mais aussi à d'autres continents (deux régions du Brésil, la Chine et le Viêt-Nam). Les contributions privilégient des entrées différentes et portent sur des périodes plus ou moins étendues, mais elles s’inscrivent toutes dans une perspective comparative - internationale, le plus souvent.

Olivier Giraud et Barbara Lucas s'interrogent sur la dynamique des "régimes de genre » en Allemagne et en Suisse dans une perspective comparative et diachronique. À partir des analyses de Sylvia Walby (2001), ils reprennent ce concept dans un sens extensif couvrant "l'ensemble des structures sociales qui influencent la division sexuée des rôles sociaux ». Ils envisagent quatre dimensions principales qui toutes contribuent à redéfinir 
les normes et les rapports de pouvoir : le rôle de l'État dans la structuration et la régulation des rapports de genre ; les arrangements concrets entre acteurs privés, associatifs et publics ; les rapports spécifiques entre sphère privée et sphère publique conduisant à telle ou telle répartition des rôles sexués ; la prise en compte de différents acteurs (associations, mouvements de femmes, syndicats, organisations patronales, partis politiques, Églises, etc.) contribuant à la transformation du régime de genre.

Ils spécifient dans un premier temps les contours du modèle traditionnel du régime de genre qui reposait, dans ces pays, sur une sexuation tranchée des rôles sociaux : schématiquement, aux hommes l'activité professionnelle et l'espace public, aux femmes l'espace domestique, les travaux y afférant et les soins aux personnes les plus faibles. À travers l'analyse des dynamiques discursives, ils montrent que, sur la base d'alliances de secteurs catholiques conservateurs et de secteurs plus marqués à gauche, le discours public dessine progressivement une nouvelle configuration des régimes de genre, avec cependant des variantes significatives selon le pays concerné. Ainsi, en Allemagne, la question du genre se construit principalement autour d'enjeux limités aux questions familiales et, dans une moindre mesure, autour du travail. En Suisse, par-delà les variations locales, le nouveau régime de genre semble s'inscrire dans le cadre d'une lutte "sexuellement neutre contre les discriminations". Dans les deux pays, le régime de genre se décline en outre différemment selon la situation sociale des ménages, les rapports de sexe s’articulant avec les rapports de classe (Pfefferkorn 2007). Le nouveau régime de genre repose désormais sur un «investissement fort de la vie publique et politique par les femmes » et sur la "conciliation » au féminin, de la vie professionnelle et de la vie familiale. Mais cette " conciliation » est loin d'aller sans contraintes et tensions pour les femmes des milieux populaires et des catégories moyennes, seules les familles aisées offrent la possibilité d'un choix moins contraint pour les femmes.

Fiona Williams, Constanza Tobío et Anna Gavanas nous proposent une analyse des 'régimes du care' qui articulent les politiques de garde des enfants en bas âge et les politiques migratoires. Ces régimes s'inscrivent dans la 'chaîne inter- 
nationale du care' qui voit des femmes venant de pays pauvres prendre en charge les enfants et le travail domestique dans les pays du Nord, tandis que leurs propres enfants sont pris en charge par d'autres femmes dans leurs pays d'origine. Cette configuration est étudiée par les auteures en s'appuyant sur des recherches qualitatives menées en Grande-Bretagne et en Espagne à la fois auprès de femmes employant ces travailleuses et de femmes immigrées à la recherche de ce type de travail. Le 'déficit du care' dans ces deux pays a pour origine les contradictions résultant de la montée du travail professionnel des femmes et les inégalités maintenues entre hommes et femmes dans l'espace domestique, mais aussi les lacunes des politiques publiques, en premier lieu l'insuffisance des structures publiques de garde d'enfant.

La marchandisation du care permet alors de lever partiellement ces contradictions grâce au travail de femmes migrantes. Les auteures montrent que le recours à ces dernières est une ressource fondamentale en Espagne pour les femmes travaillant à plein temps et ne disposant pas, à proximité, d'un réseau familial d'appui (mère, belle-mère ou grand-mère). En revanche, ce phénomène est moins marqué en Grande-Bretagne où il ne constitue qu'une solution parmi d'autres, principalement pour les femmes des catégories sociales les plus élevées. Cet article permet de montrer à quel point les changements de la situation géopolitique (Est-Ouest, Nord-Sud) et la polarisation mondiale des richesses contribuent finalement à produire une citoyenneté sexuée à un double titre. D’une part, les travailleuses migrantes peuvent certes avoir accès à des revenus, mais leurs droits sociaux, économiques, politiques et intimes ne sont pas comparables à ceux de leurs employeuses. D'autre part, cela contribue à figer les rapports sociaux de sexe et à naturaliser le travail domestique, ce dernier étant considéré comme devant être assuré par des femmes. Dans ce contexte, les tensions et rapports de pouvoir entre hommes et femmes se déplacent : les rapports entre femmes, employées et patronnes, tendent à s'aiguiser, notamment à propos des normes éducatives, tensions qui tendent par ailleurs à réactiver les stéréotypes de 'race' et de classe.

Dorottya Szikra et Dorota Szelewa s'intéressent aux politiques sociales mises en œuvre dans les pays de l'Est et à leurs effets 
sur l'articulation travail/famille. Leur réflexion s'appuie principalement sur les exemples hongrois et polonais dont elles proposent une comparaison fine, principalement sur la période des années 1950-1980. Elles mobilisent pour leur analyse des concepts élaborés dans d'autres contextes sociaux, économiques et politiques par les chercheur·e's des pays occidentaux, notamment les concepts de 'maternalisme' et de 'familialisme', tout en s'interrogeant sur la pertinence d'une telle transposition conceptuelle. Leur objectif est à la fois de contribuer à une réécriture genrée de l'histoire des politiques sociales de ces pays et de développer des cadres conceptuels originaux permettant une analyse comparative des différentes expériences nationales menées pendant et après l'époque 'socialiste'.

Le concept de 'familialisme' leur semble particulièrement adapté pour saisir les différences entre les politiques publiques mises en œuvre en Hongrie et en Pologne au regard de l'articulation vie professionnelle et vie familiale. Elles proposent une analyse détaillée des mesures mises en œuvre dans chacun des deux pays et soulignent le rôle particulier joué par l'Église catholique en Pologne. Elles montrent en substance qu'avant le tournant de la fin des années 1980, le soutien de l’État était bien plus présent en Hongrie qu'en Pologne :

Les familles hongroises avaient à leur disposition trois sortes d'allocations de congé parental et un large réseau de services d'aide à l'enfance [...]. En Pologne, où l'accès aux allocations parentales et familiales a toujours été accordé en fonction des moyens et où la fréquentation des jardins d'enfants n'a jamais dépassé $50 \%$, la situation des familles, et donc des femmes, a toujours été plus difficile.

Jacqueline Heinen propose une réflexion plus globale centrée sur les retombées sur les rapports sociaux de sexe des politiques sociales et familiales mises en œuvre dans l'Europe de l'Est depuis le tournant intervenu à la fin des années 1980. Par-delà les différences économiques ou culturelles entre les États concernés, elle insiste davantage sur les similitudes de ces politiques qui apparaissent nettement et la comparaison avec la période antérieure fait d'autant mieux ressortir les traits communs. Les droits sociaux de type universel et une certaine conception de l'égalité entre hommes et femmes sont rapidement et massivement remis 
en cause au cours des années 1990. Le tableau d'ensemble de la période antérieure, plutôt positif sur ce point en comparaison avec la période récente, doit cependant être nuancé. En effet, la réduction du rôle des femmes à celui de mères confortait des représentations et des pratiques sociales inégalitaires (ségrégation sexuée de l'emploi, écart de salaires en faveur des hommes, faible partage du travail domestique, double journée de travail pour les femmes). Ces contradictions passées ont laissé des traces et expliquent aussi partiellement la tendance récente au repli sur l'espace domestique.

À partir des années 1990, les mesures protectrices ciblant les mères ou les parents de jeunes enfants, comme plus largement les politiques sociales d'ensemble, sont mises à mal : baisse du montant des allocations, réduction des congés parentaux, diminution du nombre de places dans les structures de garde des jeunes enfants, etc. La montée du chômage, la baisse des revenus et les difficultés matérielles rencontrées par la majorité des ménages de ces pays accentuent en outre l'idéalisation du rôle de la famille et la valorisation de celui des mères. Dans un contexte de dégradation de la situation sociale, les inégalités de sexe ont alors tendance à passer au second plan et la rhétorique et les pratiques sexistes, comme les politiques publiques inégalitaires, trouvent à s'exprimer plus ouvertement, conduisant notamment à la montée des violences envers les femmes, à la féminisation de la pauvreté et à l'effondrement de la natalité. Le déficit démographique est cependant à envisager autant comme le résultat de la précarisation sociale des femmes que la conséquence de leur volonté de s'accrocher à leur emploi, car comme le souligne Jacqueline Heinen :

La chute radicale du nombre de naissances constitue un phénomène qui peut être interprété comme un signe de résistance des jeunes femmes confrontées à des difficultés financières sans précédent.

Isabelle Attané et Catherine Scornet s'intéressent aux relations entre la condition des femmes et les politiques reproductives mises en œuvre en Chine et au Viêt-Nam au cours des dernières décennies. Elles comparent les deux pays au cours de deux périodes : les décennies 1950 à 1970 qui voient les deux pays tenter de contrôler la croissance de leur population pour 
amorcer un développement économique dans une perspective socialiste ; et les vingt ou trente dernières années marquées par des bouleversements économiques et sociaux considérables dans le contexte de la libéralisation économique. Les auteures montrent qu'en dépit d'éléments favorables à l'amélioration du statut des femmes (lois favorables à l'égalité, scolarisation primaire de masse, baisse de la fécondité, taux d'activité élevés), la spécification des rôles de sexe persiste et les obstacles à leur émancipation demeurent. La baisse de la fécondité a eu un impact direct très limité sur l'activité professionnelle des femmes car avant même que la fécondité ne baisse, c'est-à-dire jusqu'au tout début des années 1970, l'activité féminine était déjà extrêmement développée, et il était donc difficile de la faire augmenter davantage.

Le contexte de la libéralisation économique est venu contrebalancer les effets potentiellement positifs d'une réduction de la fécondité en réactivant les rôles sociaux traditionnels et en bridant la dynamique d'autonomisation des femmes: la libéralisation du marché du travail s'est traduite dans certaines régions par une baisse de l'activité féminine ; le désengagement partiel de l'État des entreprises et des structures éducatives et sanitaires a eu des effets analogues, en particulier au Viêt-Nam ; la fin de la gratuité des crèches et de l'école a contraint de nombreuses femmes à quitter leur emploi pour s'occuper de leurs enfants ; les frais de scolarité et les dépenses de santé ne sont plus assumés entièrement par l'État. Les femmes sont de plus en plus nombreuses à devoir choisir entre maternité et activité professionnelle. Et les auteures de conclure :

Si les fondements de l'émancipation des femmes ont pu être jetés dans chacun des deux pays au cours du demi-siècle écoulé, il n'en reste pas moins qu'ils sont fragiles car soumis à des forces contradictoires.

Enfin, la contribution de Bila Sorj et Adriana Fontes examine l'impact de deux politiques publiques sur l'articulation entre travail et famille : le Programme Bolsa Familia (Bourse Famille), mis en œuvre par le gouvernement brésilien pour lutter contre la pauvreté, s'adresse aux femmes en tant que mères ; la politique générale de la petite enfance qui donne accès aux enfants de moins de six ans aux crèches et aux écoles mater- 
nelles concerne les mères en tant que travailleuses. Les effets de ces politiques sont étudiés en comparant les deux régions du pays les plus peuplées : le Nordeste et le Sudeste. Le contraste entre les deux est important puisque la seconde est deux fois plus riche, le pourcentage de pauvres y est trois fois moins élevé et l'analphabétisme nettement moins répandu. La Bourse Famille a clairement un impact négatif sur l'activité professionnelle des femmes, plus marqué dans le Nordeste que dans le Sudeste. Cette politique de lutte contre la pauvreté est manifestement entachée de maternalisme. Inversement, l'accès accru aux services d'accueil à la petite enfance amène plus fréquemment les mères pauvres sur le marché du travail, surtout dans le Sudeste, en raison de la plus grande marchandisation des activités de care.

Cette étude confirme les résultats des expériences européennes : l'accès aux crèches ou aux maternelles et à des écoles à plein temps, de même que l'extension du congé paternité, contribuent à l'entrée des femmes sur le marché du travail et, par la suite, amènent à questionner la naturalité du lien entre les femmes et le travail domestique. Inversement, elle montre aussi qu'en faisant de l'attribution des tâches familiales aux femmes l'objet d'une politique publique, le Programme Bourse Famille joue surtout en sens inverse, renforce la division sexuelle du travail, affaiblit la relation des femmes avec le monde du travail et ne contribue que très partiellement à la promotion de l'autonomie des femmes :

Recevoir ces avantages signifie pour les femmes bénéficiaires une extension de leur maternage, c'est-à-dire de leur rôle auprès des enfants en qualité de mère ou de substitut (Paez-Souza et Vaitsman 2007, citées par Sorj et Fontes).

Le panorama d'ensemble qui se dégage de ce numéro atteste les nombreuses variations qui se dégagent d'un pays à l'autre selon : le poids des structures étatiques et le rôle des Églises ; les visées plus ou moins interventionnistes des acteurs politiques, notamment en matière de politiques reproductives ; l'ampleur des discriminations socioéconomiques à l'œuvre ; la part des tâches de care dévolues à des personnes extérieures à la famille, notamment à des immigrées, etc. Mais il atteste également un trait commun à tous les pays considérés quant aux 
responsabilités intrafamiliales. Par-delà les efforts consentis dans tel ou tel pays pour promouvoir l'autonomie économique des femmes, se dégage en effet la persistance - à des degrés variables, il est vrai - de pratiques sexuées en matière de care et de prise en charge des jeunes enfants. Et ces inégalités sont d'autant plus sensibles qu'il s'agit de femmes migrantes ou issues des catégories sociales défavorisées. La dimension comparative des études menées a ceci d'heuristique qu'elle donne un aperçu de l'ampleur des changements indispensables, sur le plan politique comme sur celui des représentations, pour que les politiques familiales s'adressent aux hommes autant qu'aux femmes et pour que la mixité s'impose enfin dans les pratiques. Ce numéro des Cahiers du Genre, comparant ces politiques entre pays contrastés du Sud, de l'Est et du Nord, veut contribuer à la controverse en cours.

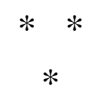

L’article de Virginie Rozée, publié en hors-champ, examine l'exercice des droits reproductifs et sexuels des femmes boliviennes. L'auteure montre qu'en dépit des mesures instaurées par les gouvernements successifs en faveur des droits des femmes, leur application se heurte à de nombreux obstacles. Elle souligne notamment que la décision pour les femmes d'une pratique contraceptive reste du ressort des hommes. Elle s'attache à décortiquer les mécanismes qui, dans une société fortement marquée par le catholicisme et la domination masculine, font perdurer l'image de la madresposa, cette mèreépouse qui ne s'épanouirait que dans la maternité et ce, au détriment de sa santé.

Notre rubrique « Lecture d'une œuvre » fait place, cette fois, à la pensée de la philosophe féministe américaine Iris Marion Young, disparue en 2006. Marie Garrau et Alice Le Goff montrent comment l'œuvre de Young articule critique sociale et phénoménologie et ouvre la voie à une pensée originale du sujet collectif du féminisme. Par son concept de 'solidarité différenciée', elle souligne que nous sommes toujours pris·e·s dans des relations multiples d'interdépendance de natures et de niveaux différents. Cette conception pluraliste de l'espace 
public l'amène à réviser l'idée d'inclusion politique grâce à la prise en compte de l'articulation des différences sociales, culturelles, économiques, géographiques, etc., quitte à voir s'éloigner un idéal d’intégration.

Jacqueline Heinen, Helena Hirata et Roland Pfefferkorn

\section{Références}

Dauphin Sandrine (2008). «Conciliation travail-famille : du côté des entreprises ». Recherches et prévisions, $n^{\circ}$ 92, juin.

Fagnani Jeanne, Letablier Marie-Thérèse (2001). « Travail et famille : contraintes et arbitrages ». Problèmes politiques et sociaux, $\mathrm{n}^{\circ} 858$ « Famille et travail : contraintes et arbitrages ».

Fouquet Annie (2005). «Les politiques d'emploi envers les femmes ». In Maruani Margaret (ed). Femmes, genre et sociétés. L'état des savoirs. Paris, La Découverte.

Gautier Arlette, Heinen Jacqueline (eds) (1993). Le sexe des politiques sociales. Paris, Côté-femmes.

Heinen Jacqueline (2000). « Politiques sociales et familiales ». In Hirata Helena, Laborie Françoise, Le Doaré Hélène, Senotier Danièle. Dictionnaire critique du féminisme. Paris, PUF [ $2^{\mathrm{e}}$ éd. 2004].

Hiroki Sato (2008). "Amélioration du taux d'emploi des femmes et réalisation d'une société privilégiant le 'work life balance'». In Promotion du travail des femmes et conciliation de la vie professionnelle et familiale. Séminaire franco-japonais, Paris, 19-20 novembre 2007, Délégation aux affaires européennes et internationales, ministère du Travail et de la Santé, Paris, CDRom.

Junter-Loiseau Annie (1999). «La notion de conciliation de la vie professionnelle et de la vie familiale : révolution temporelle ou métaphore des discriminations ? » Cahiers du genre, $\mathrm{n}^{\circ} 24$.

Leira Arnlaug (2002). Working Parents and the Welfare State: Family Change and Policy Reform in Scandinavia. Cambridge, Cambridge University Press.

Lister Ruth et al. (2007). Gendering Citizenship in Western Europe: New Challenges for Citizenship Research in a Cross-National Context. Bristol, The Policy Press.

Pfefferkorn Roland (2007). Inégalités et rapports sociaux : rapports de classes, rapports de sexes. Paris, La Dispute « Le genre du monde ». 
Walby Sylvia (2001). "From Gendered Welfare State to Gender Regimes: National Differences, Convergence or Re-structuring?” Paper presented to Gender and Society Group, Stockholm.

Williams Fiona (2004). Rethinking Families. London, Calouste Gulbenkian Foundation. 